05,13

\title{
Влияние ориентации магнитного момента зонда магнитно-резонансного силового микроскопа на спектры спин-волновых резонансов
}

\author{
(C) Е.В. Скороходов ${ }^{1}$, М.В. Сапожников ${ }^{1,2}$, Р.В. Горев ${ }^{1}$, \\ А.П. Володин ${ }^{3}$, В.Л. Миронов ${ }^{1,2}$ \\ ${ }^{1}$ Институт ффизики микроструктур РАН, \\ Нижний Новгород, Россия \\ ${ }^{2}$ Нижегородский государственный университет им. Н.И. Лобачевского, \\ Нижний Новгород, Россия \\ ${ }^{3}$ KU Leuven, Afdeling Vaste-stoffysica en Magnetisme, Celestijnenlaan 200D, \\ Leuven, Belgium \\ E-mail: evgeny@ipmras.ru
}

Проведены исследования ферромагнитного резонанса в массиве микрополосок пермаллоя размером $3000 \times 500 \times 30 \mathrm{~nm}^{3}$, упорядоченных на прямоугольной решетке $3.5 \times 6 \mu \mathrm{m}^{2}$, методом магнитнорезонансной силовой микроскопии в условиях сильного взаимодействия зонда с образцом. Это взаимодействие приводит к сложным модификациям наблюдаемых спектров, выражаюшимся как в расщеплении линий, так и в изменениях формы спектров. Анализируются зависимости полученных спектров от расстояния зондобразец и ориентации магнитного момента зонда.

Работа выполнена при поддержке Российского научного фонда (проект 16-12-10254).

DOI: $10.21883 /$ FTT.2018.11.46665.25NN

\section{1. Введение}

В последние годы интенсивное развитие получил новый метод исследования СВЧ свойств ферромагнитных наноструктур с высоким пространственным разрешением - магнитно-резонансная силовая микроскопия (МРСМ). Данный метод основан на детектировании высокочастотных колебаний намагниченности образца с помощью чувствительного механического датчика в виде упругой консоли малой жесткости (кантилевера) с магнитным зондом на конце [1-9]. Схема экспериментальной реализации метода МРСМ показана на рис. 1. В МРСМ производится накачка образца СВЧ-полем $\mathbf{h}$, модулированным по амплитуде, на резонансной частоте механических колебаний кантилевера.

Принцип работы МРСМ основан на том, что магнитный зонд является механическим детектором с полосой детектирования в килогерцовой области. В условиях СВЧ-накачки, промодулированной на частоте собственных колебаний кантилевера, амплитуда его колебаний пропорциональна высокочастотной магнитной восприимчивости образца. Тем самым это позволяет измерять спектры ферромагнитного резонанса (ФМР). Подробнее метод МРСМ описан ниже. При этом МРСМ-спектры регистрируются в виде зависимости амплитуды или фазы колебаний кантилевера от частоты СВЧ-накачки или от внешнего магнитного поля. МРСМ-изображения регистрируются при фиксированной частоте накачки посредством сканирования зондом над поверхностью образца. Таким образом, МРСМ-изображение представляет собой распределение амплитуды вынужденных колебаний кантилевера в зависимости от положения зонда над образцом, что соответствует распределению амплитуды колебаний намагниченности по образцу на заданной частоте СВЧ-поля. Пространственное разрешение МРСМ определяется областью магнитостатического взаимодействия зонда с образцом и шириной линии магнитного резонанса.

В ферромагнетиках, из-за сильного обменного взаимодействия, поглощение СВЧ-излучения приводит к возбуждению коллективных колебаний намагниченности - спиновых волн, которые могут быть исследованы методом МРСМ. Диапазон частот ферромагнитного резонанса для металлических ферромагнетиков лежит в области выше $0.1 \mathrm{GHz}$. При этом эффективным методом модификации спектра поглощения и пространственных мод спин-волновых резонансов является наноструктурирование образцов [10-12].

В настоящей работе приводятся результаты МРСМисследований ФМР в массиве микрополосок пермаллоя в продольном подмагничивающем поле. Известно, что зонд может существенно влиять на равновесное распре-

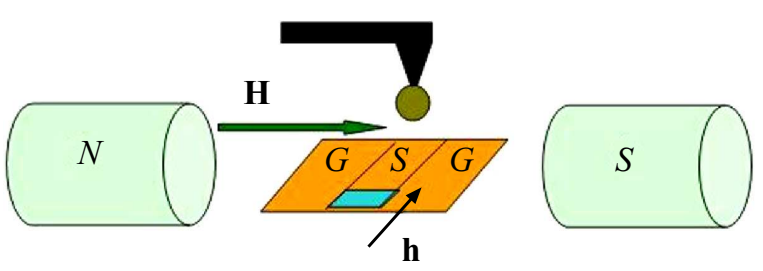

Рис. 1. Геометрия проведения МРСМ-измерений. 
деление намагниченности, а, следовательно, оказывать воздействие и на спектры ФМР [13-15], поэтому в настоящей работе особое внимание уделяется детальному исследованию этого воздействия.

\section{2. МРСМ-исследования массива микрополосок пермаллоя}

Нами были проведены МРСМ-исследования ФМРмассива планарных микрополосок пермаллоя $\mathrm{Ni}_{80} \mathrm{Fe}_{20}$ (далее обозначается как $\mathrm{NiFe}$ ), изготовленных методом взрывной (lift-off) литографии [16-18].

СЭМ-изображение участка массива микрополосок приведено на рис. 2. Полоски имеют размеры $3000 \times 500 \times 30 \mathrm{~nm}^{3}$ и упорядочены на прямоугольной решетке с периодом $6 \mu \mathrm{m}$ в направлении длинной оси и $3.5 \mu \mathrm{m}$ в поперечном направлении.

Измерения проводились с использованием магнитнорезонансного силового микроскопа [19]. В качестве зондового датчика использовался стандартный кантилевер NSG-1 (НТ-МДТ), на который наклеивалась магнитная частица $\mathrm{CoSm}$ с характерным диаметром $10 \mu \mathrm{m}$. Образец располагался на планарной полосковой линии так, что магнитная составляющая СВЧ-поля накачки была направлена вдоль короткой оси полосок. При этом внешнее подмагничивающее поле Н было ориентировано вдоль длинной оси полосок. В качестве МРСМ-спектров регистрировались зависимости амплитуды колебаний кантилевера от внешнего подмагничивающего поля. Измерения проводились при различных расстояниях между магнитным зондом и образцом $L$. Накачка образца производилась на частоте $f=5.8 \mathrm{GHz}$ с мощностью $20 \mathrm{dBm}$. СВЧ-поле модулировалось по амплитуде на резонансной частоте кантилевера. Измерения проводились в вакууме $\left(10^{-3}\right.$ Torr $)$. В этом случае добротность зонда $Q$ составляла 1000 . Получение МРСМ-спектров осуществлялось при комнатной температуре.

Сила магнитного взаимодействия между зондом и образцом, вызывающая колебания кантилевера, имеет вид

$$
\mathbf{F}=-\nabla \int_{V_{\text {sample }}}\left(\mathbf{m h}_{p}\right) d V
$$

где $\mathbf{m}$ - квазистатическая компонента намагниченности образца, которая при СВЧ накачке осциллирует с частотой, равной резонансной частоте кантилевера, $\mathbf{h}-$ магнитное поле зонда в области образца, интегрирование проводится по объему образца $V_{\text {sample. }}$ Раскачка кантилевера происходит за счет z-компоненты силы, которая в случае однородно намагниченного образца имеет вид

$$
F_{z}=-\int_{V_{\text {sample }}}\left(m_{x} \frac{\partial h_{p x}}{\partial z}+m_{y} \frac{\partial h_{p y}}{\partial z}+m_{z} \frac{\partial h_{p z}}{\partial z}\right) d V .
$$

Видно, что данная сила существенно зависит от степени неоднородности поля зонда в области образца. Обратим

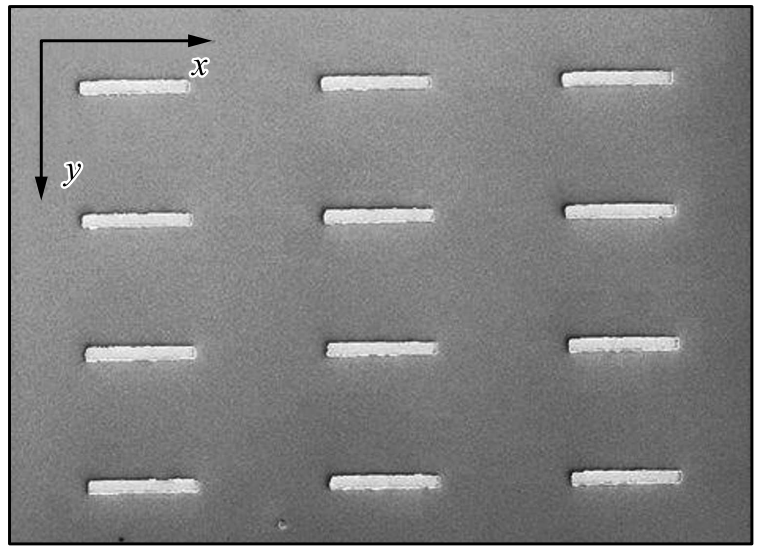

Рис. 2. СЭМ-изображение участка массива микрополосок $\mathrm{NiFe}$. Размеры полосок $3000 \times 500 \mathrm{~nm}^{2}$.

внимание на то, что знак силы противоположен знаку градиента магнитного поля. В простейшей модели зонд можно представить в виде однородно намагниченного шара с магнитным моментом $\mu$, равным

$$
\mu=\frac{4}{3} \pi R^{3} M_{s}
$$

где $R$ - радиус шара, $M_{s}-$ намагниченность насыщения материала. Такой зонд создает в окружающем пространстве поле, соответствующее полю точечного магнитного диполя, расположенного в центре шара

$$
\mathbf{h}=\frac{3 \mathbf{r}(\mathbf{r} \boldsymbol{\mu})}{r^{3}}-\frac{\boldsymbol{\mu}}{r}
$$

Это поле существенно неоднородно и анизотропно, поэтому характер взаимодействия зонда и образца в МРСМ сильно зависит от направления магнитного момента зонда по отношению к намагниченности образца.

Необходимо заметить, что наличие СВЧ-поля в рабочем промежутке микроскопа приводит к появлению немагнитного взаимодействия (сила $F_{\text {nonmagnetic }}$ ) между образцом и кантилевером, обусловленного образованием разности потенциалов между копланарной линией и зондом, токами Фуко, нагреванием зонда [20]. Поскольку СВЧ-сигнал промодулирован по амплитуде, то данное взаимодействие также может привести к резонансной раскачке кантилевера. Таким образом, результирующая сила, действующая на зонд, является суммой немагнитной и магнитной сил

$$
\mathbf{F}=\mathbf{F}_{\text {nonmagnetic }}+\mathbf{F}_{\text {magnetic }} .
$$

С другой стороны, сила магнитного взаимодействия между образцом и магнитным зондом зависит от величины внешнего поля и возникает лишь в условиях возбуждения ФМР, в то время как немагнитная сила постоянна. В результате при сканировании по величине внешнего магнитного поля ФМР-спектр будет виден на 


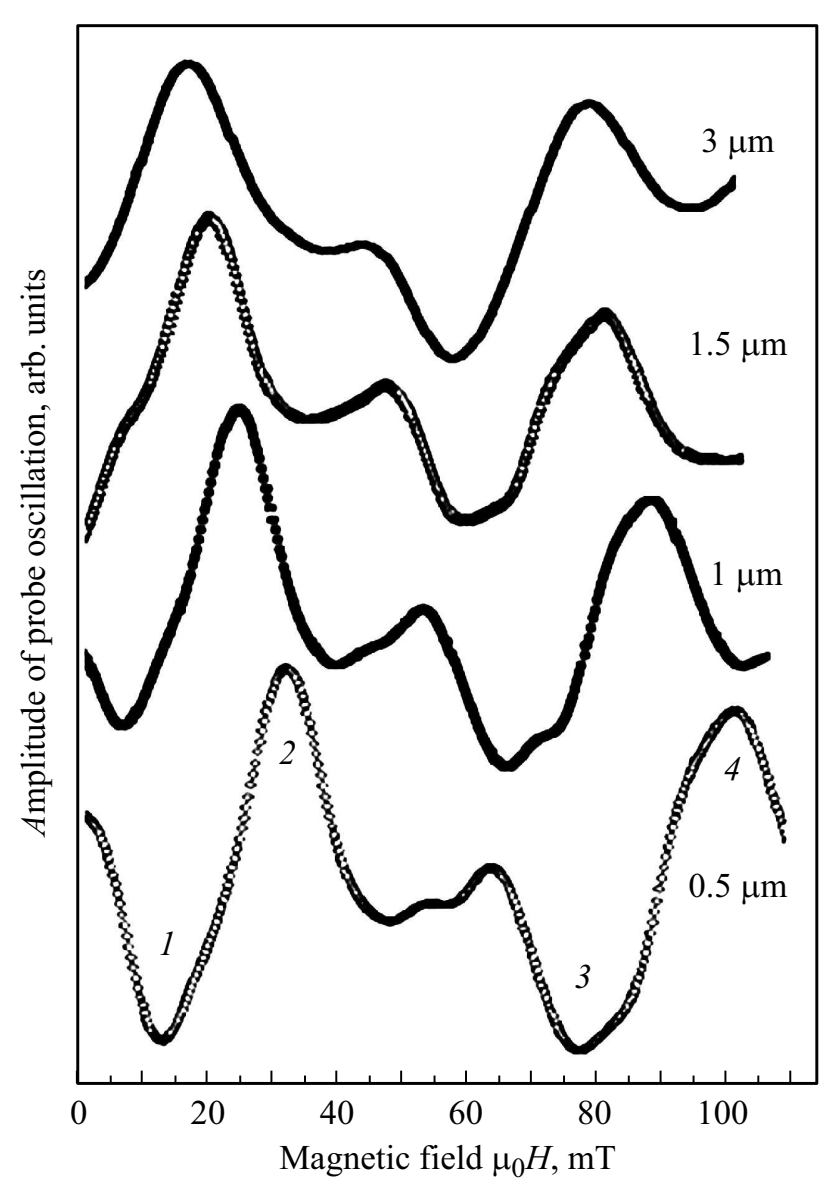

Рис. 3. МРСМ-спектры ФМР-микрополосок NiFe для случая, зонда, намагниченного перпендикулярно поверхности образца, зарегистрированные при расстояниях между зондом и образцом равных $0.5,1,1.5$ и $3 \mu \mathrm{m}$. Спектры сдвинуты по вертикальной оси для удобства сравнения. Наблюдаемые резонансы пронумерованы.

фоне постоянного сигнала, связанного с возбуждением колебаний кантилевера немагнитного происхождения [20]. Соответственно в случае, если возникающая в области ФМР магнитная сила (2) совпадает по фазе с немагнитной силой, в спектре будут наблюдаться пики, а в случае если магнитная и немагнитная силы противоположны по фазе, то в спектре будут наблюдаться провалы. Сдвиг фазы магнитной силы относительно немагнитной зависит от знака градиента магнитного поля зонда в области образца и меняется на $180^{\circ}$ при изменении знака градиента.

В первой серии экспериментов МРСМ-измерения проводились с применением зонда, у которого магнитный момент был направлен по оси $z$, то есть вдоль нормали к поверхности образца. Зонд располагался над центральной областью одной из микрополосок. Магнитная частица $\mathrm{CoSm}$ зонда обладает большой коэрцитивностью (более $2.5 \mathrm{~T}$ ). Это означает, что магнитный момент частицы сохраняет направление, независимо от направлений и величин внешних магнитных полей, которые используются в эксперименте. На рис. 3 приведены МРСМ-спектры, полученные для расстояний между зондом и образцом $L=0.5,1,1.5,3$ мкм. Как видно из рисунка, при больших $L$ в спектре МРСМ наблюдается два выраженных пика в области полей 30 и $100 \mathrm{mT}$ и два провала в области полей 10 и $80 \mathrm{mT}$ (для $L=0.5 \mu \mathrm{m})$.

Сложная форма спектра связана с тем, что подмагничивающее поле, в котором находится микрополоска и величина которого определяет условия ФМР, складывается из внешнего поля и поля магнитного зонда. Рассмотрим пять ближайших к зонду микрополосок непосредственно под зондом и по две микрополоски по короткой и длинной осям, которые, по всей видимости, и дают основной вклад в наблюдаемый спектр. Поле зонда имеет разную величину и направление в разных областях образца. При этом надо выделить три существенных для понимания формы спектра эффекта: 1) вертикальная составляющая поля зонда в любой точке образца направлена поперек оси магнитной анизотропии полосок, обусловленной их формой, и поэтому ее наличие сдвигает ферромагнитный резонанс в область сильных полей (см. табл. 1.1 из [21]); 2) горизонтальная составляющая поля зонда $\mathbf{h}_{p k}$ уменьшает поле резонанса, если она сонаправлена внешнему магнитному полю, и увеличивает его величину, если она противоположна ему; 3) градиент магнитного поля, определяющий знак магнитной силы, может быть разным в разных областях образца, соответственно ФМР этих областей может проявляться в спектре как в виде пика, так и в виде провала.

Проанализируем экспериментально наблюдаемый МРСМ-спектр (рис. 3). На спектре резонансы видны парами, (1-2) и (3-4). Каждая пара представляет собой провал с последующим пиком. Первая пара резонансов соответствует спин-волновым возбуждениям. Низкополевые пик и провал (1 и 2, рис. 2) включают

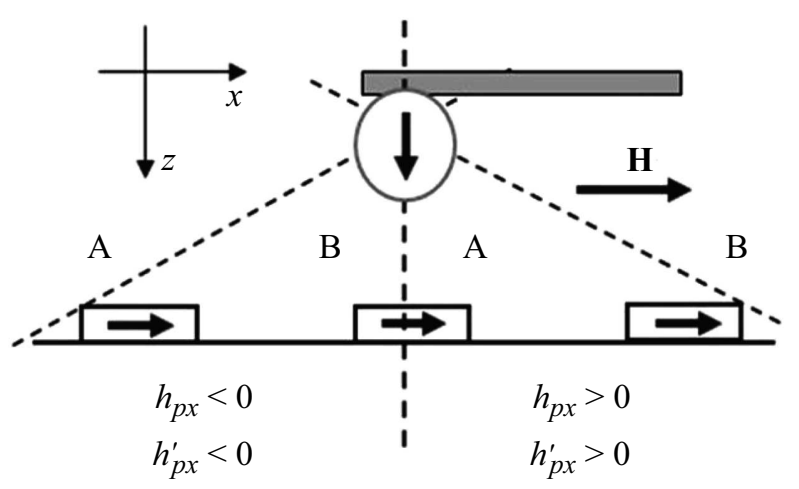

Рис. 4. Геометрия эксперимента. Магнитный момент зонда направлен перпендикулярно поверхности образца. Пунктирными линиями показано сечение конических областей, различающихся знаками проекций магнитного поля зонда и его градиента на ось $x$. 


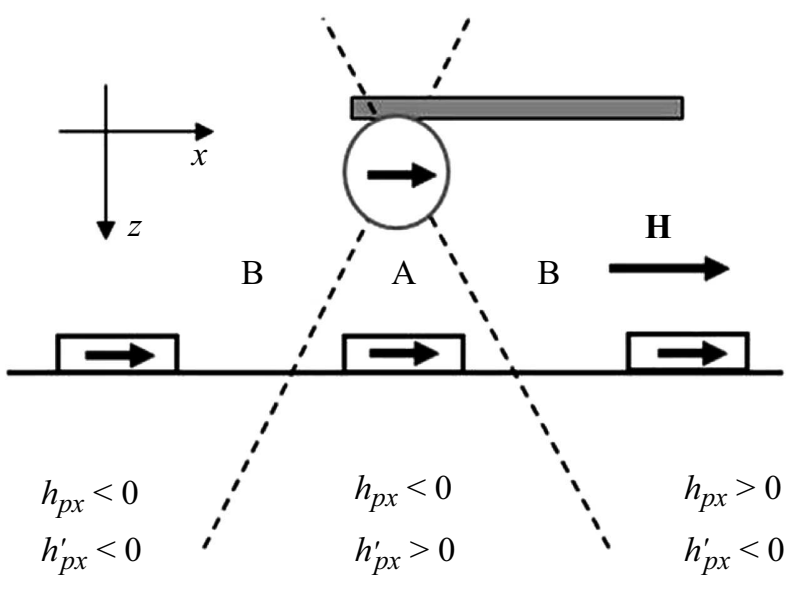

Рис. 5. Геометрия эксперимента. Магнитный момент зонда направлен вдоль плоскости образца. Пунктирными линиями показано сечение конических областей, различающихся знаками проекций магнитного поля зонда и его градиента на ось $x$.

в себя резонансы, обусловленные возбуждением спинволновых мод. Вторая пара высокополевых резонансов (3 и 4, рис. 3) соответствует краевым модам. Расщепление внутри пары резонансов можно понять из рис. 4. Величина и направление поля зонда $\mathbf{h}_{p x}$, a также знак градиента поля $h_{p x}^{\prime}=\frac{\partial h_{p x}}{\partial z}$ имеют различные значения в областях А и В. В полосках, которые лежат в области $\mathrm{A}$, поле $\mathbf{h}_{p x}$ сонаправлено внешнему полю электромагнита, а его градиент положителен. Поэтому данные резонансы имеют форму провала и сдвинуты в область слабых полей. В полосках, которые лежат в области $\mathrm{B}$, ситуация противоположная: поле $\mathbf{h}_{p x}$ направлено противоположно полю электромагнита, a градиент поля отрицателен. Поэтому резонансы имеют форму пика и сдвинуты в область более сильных полей. Таким образом, разница в направлении $\mathbf{h}_{p x}$ приводит к расщеплению резонансов, разница в знаках градиента $\mathbf{h}_{p x}$ приводит к тому, что один резонанс проявляется в виде пика, другой - в виде провала. Приближение зонда к образцу приводит к увеличению $\mathbf{h}_{p x}$ во всех областях образца, что приводит к наблюдаемому смещению всего спектра как целого в область более сильных полей. Границы областей А и В определяются из условия

$$
\frac{\partial h_{p x}}{\partial z}=0 \text {. }
$$

Отсюда следует, что связь между высотой $d$, на которой расположен зонд, и координатой $x$ границы области на поверхности образца будет иметь следующий вид:

$$
x=\frac{d}{\sqrt{2}} .
$$

В том случае, когда магнитный момент зонда направлен вдоль поверхности образца (рис. 5), регистрируемые МРСМ-спектры (рис. 6) имеют существенно другую форму. Это связано с изменением пространственной структуры поля и градиента поля, создаваемого магнитным зондом. В этом случае пространство над образцом разбивается на три области с различным знаком продольной компоненты градиента поля $\mathbf{h}_{p x}$.

Из рис. 6 видно, что когда зонд располагается высоко над образцом $(L=3$ мкм), в спектре наблюдаются широкий провал, который соответствует нескольким резонансным возбуждениям вблизи значений полей 35 и $47 \mathrm{mT}(1,2,3$, рис. 6) и провал около $85 \mathrm{mT}(4$, рис. 6$)$. При уменьшении расстояния $L$ до $1.5 \mu \mathrm{m}$ наблюдается выраженное разрешение близко расположенных резонансных возбуждений 1,2 (в области $35 \mathrm{mT})$ и $3(65 \mathrm{mT}$, см. рис. 6). По-видимому, провалы 1 и 2 на кривой при $L=3 \mu \mathrm{m}$ соответствуют резонансам, связанным со спин-волновыми колебаниями, а резонансы 3 и 4 с краевыми модами [16]. Разброс расстояний между зондом и полосками приводит к разбросу резонансных магнитных полей в массиве, что в свою очередь приводит к уширению провалов на экспериментальных

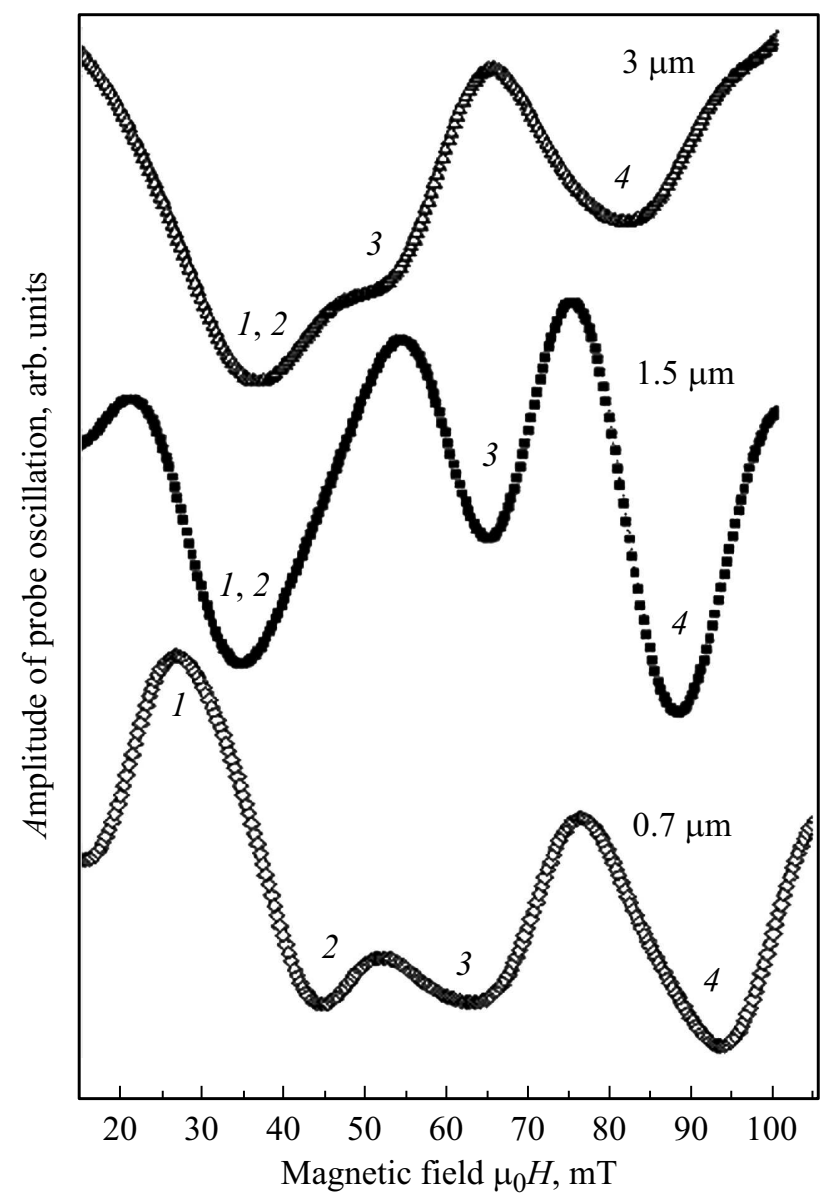

Рис. 6. МРСМ-спектры ФМР-микрополосок NiFe для случая, когда зонд намагничен вдоль поверхности образца, зарегистрированные при различных расстояниях между зондом и образцом, равных (снизу вверх) 0.5, 1 и $3 \mu \mathrm{m}$. Спектры сдвинуты по вертикальной оси для удобства сравнения. Наблюдаемые резонансы пронумерованы. 


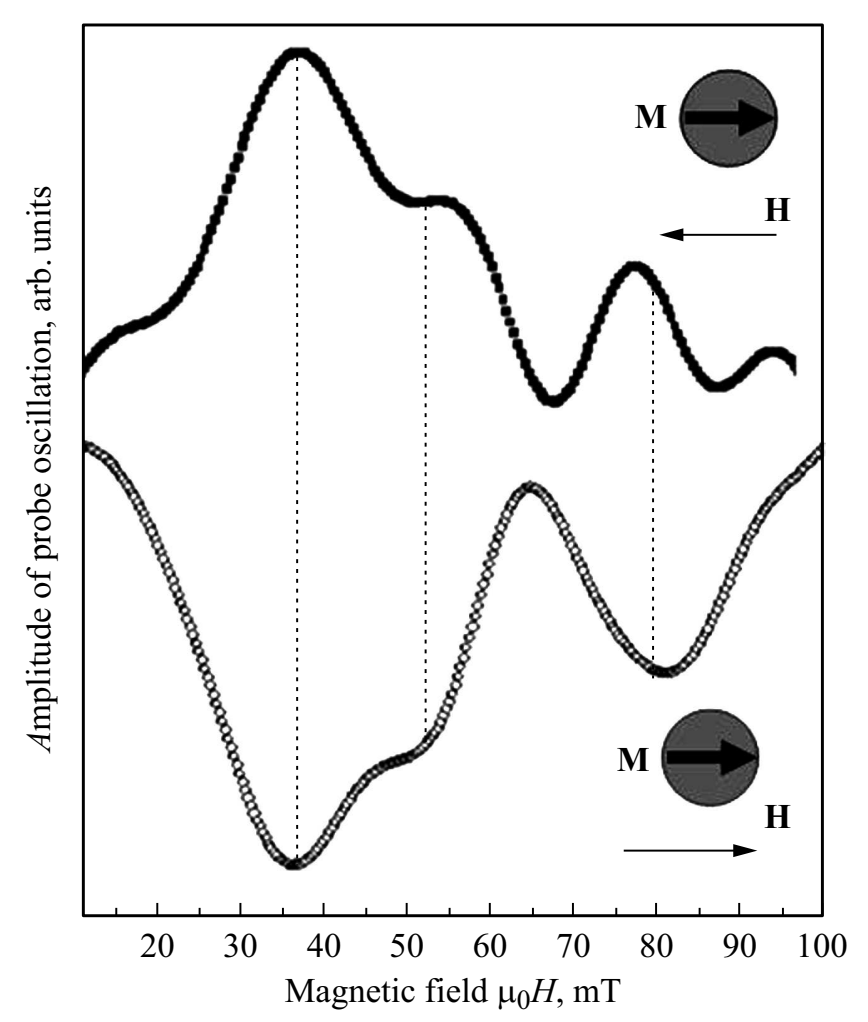

Рис. 7. МРСМ-спектры ФМР-микрополосок NiFe для случая, когда зонд намагничен вдоль поверхности образца. Внешнее магнитное поле направлено так же, как и магнитный момент зонда (светлые символы), и в противоположную сторону (темные символы). Расстояние между зондом и поверхностью образца составляет $3 \mu \mathrm{m}$.

данных. Наличие только провалов связано с тем, что частицы, которые дают основной вклад в МРСМ-сигнал, находятся в области А, где градиент поля зонда положителен. При дальнейшем уменьшении $L$ полоски, расположенные вдоль длинной оси массива (ось $x$, рис. 1), попадают в области поля зонда с противоположным направлением проекции градиента поля $\mathbf{h}_{p x}$ (область В). Это приводит к тому, что связанный с ними резонанс (1, рис. 6), начинает проявляться в форме пика в поле 27 mT. В данной экспериментальной конфигурации связь между высотой $d$ и координатой $x$ границы области А на поверхности образца будет иметь следующий вид:

$$
x= \pm \frac{d}{2}
$$

На рис. 7 приведены МРСМ-спектры для случаев, когда внешнее магнитное поле сонаправлено и противоположно направлено магнитному моменту зонда. Зонд расположен на расстоянии $3 \mu \mathrm{m}$ и намагничен по оси $x$. Смена направления внешнего магнитного поля относительно магнитного момента зонда ведет к смене провалов на пики и наоборот, в соответствии с формулой (2).

\section{3. Заключение}

Таким образом, в работе приведены результаты МРСМ-исследований массивов микрополосок $\mathrm{NiFe} \mathrm{c}$ анизотропией типа легкая плоскость. Показана возможность локальной регистрации ФМР-спектров. Продемонстрирована зависимость МРСМ-спектров от направления магнитного момента зонда и внешнего магнитного поля.

Авторы выражают благодарность В.А. Быкову и В.В. Полякову за консультации и А.Н. Резнику за помощь в работе и полезные обсуждения.

\section{Список литературы}

[1] J.A. Sidles. Appl. Phys. Lett. 58, 2854 (1991).

[2] D. Rugar, R. Budakian, H.J. Mamin, B.W. Chui. Nature 403, 329 (2004).

[3] C.L. Degen, M. Poggio, H.J. Mamin, C.T. Rettner, D. Rugar. Nanoscale magnetic resonance imaging. Proceedings of the National Academy of Sciences of the USA 106, 1313 (2009).

[4] Z. Zang, P.C. Hammel, P.E. Wigen. Appl. Phys. Lett. 68, 2005 (1996).

[5] G. De Loubens V.V. Naletov, O. Klein, J. Ben Youssef, F. Boust, N. Vukadinovic. Phys. Rev. Lett. 98, 127601 (2007).

[6] O. Klein, G. de Loubens, V.V. Naletov, F. Boust, T. Guillet, H. Hurdequint, A. Leksikov, A.N. Slavin, V.S. Tiberkevich, N. Vukadinovic. Phys. Rev. B 78, 144410 (2008).

[7] H.-J. Chia, F.Guo, L.M. Belova, D. McMichael. Phys. Rev. B 86, 184406 (2012).

[8] F. Guo, L.M. Belova, D. McMichael. Phys. Rev. Lett. 110, 017601 (2013).

[9] Yu. Obukhov, D.V. Pelekhov, J. Kim, P. Banerjee, I. Martin, E. Nazaretski, R. Movshovich, S. An, T.J. Gramila, S. Batra, P.C. Hammel. Phys. Rev. Lett. 100, 197601 (2008).

[10] G.R. Aranda, G.N. Kakazei, J. González, K.Y. Guslienko. J. Appl. Phys. 116, 093908 (2014).

[11] M.V. Sapozhnikov, L.I. Budarin, E.S. Demidov. J. Magn. Magn. Mater. 449, 68 (2018).

[12] M.V. Sapozhnikov, R.V. Gorev, E.A. Karashtin, V.L. Mironov. J. Magn. Magn. Mater. 446, 1 (2018).

[13] В.Л. Миронов, А.А. Фраерман, Б.А. Грибков, О.Л. Ермолаева, А.Ю. Климов, С.А. Гусев, И.М. Нефедов, И.А. Шерешевский. Физика металлов и металловедение 110, 7, 708.

[14] V.L. Mironov, B.A. Gribkov, D.S. Nikitushkin, S.A. Gusev, S.V. Gaponov, A.B. Shubin, P.A. Zhdan, C. Binns. IEEE Trans. Magn. 44, 10, 2296 (2008).

[15] I. Lee, Yu. Obukhov, G. Xiang, A. Hauser, F. Yang, P. Banerjee, D.V. Pelekhov, P.C. Hammel. Nature 466, 845 (2010).

[16] E.V. Skorohodov, R.V. Gorev, R.R. Yakubov, E.S. Demidov, Yu.V. Khivintsev, Yu.A. Filimonov, V.L. Mironov. J. Magn. Magn. Mater. 424, 118 (2017).

[17] V.L. Krutyanskiy, I.A. Kolmychek, B.A. Gribkov, E.A. Karashtin, E.V. Skorohodov, T.V. Murzina. Phys. Rev. B 88, 9, 094424 (2013). 
[18] O.G. Udalov, M.V. Sapozhnikov, A. Gribkov, E.A. Karashtin, E.V. Skorohodov, V.V. Rogov, A.Yu. Klimov, A.A. Fraerman. Phys. Rev. B 86, 9, 94416 (2012).

[19] Е.В. Скороходов, М.В. Сапожников, А.Н. Резник, В.В. Поляков, В.А. Быков, А.П. Володин, В.Л. Миронов. Приборы и техника эксперимента. В печати.

[20] Han-Jong Chia, Feng Guo, L.M. Belova, R.D. McMichael. Phys. Rev. Lett. 108, 087206 (2012).

[21] А.Г. Гуревич, Г.А. Мелков. Магнитные колебания и волны. Наука, М. (1994).

Редактор Е.Ю. Флегонтова 